\title{
Advantages of indwelling pleural catheters for management of malignant pleural effusions
}

\author{
Rogier C. Boshuizen • Rajesh Thomas • Y. C. Gary Lee
}

Published online: 8 March 2013

(C) Springer Science+Business Media New York 2013

\begin{abstract}
The use of indwelling pleural catheters (IPC) has an established place in the management of malignant pleural effusion (MPE) when pleurodesis has failed or is unsuitable. The use of IPC as a frontline therapy in place of pleurodesis is also gathering momentum. Recent studies confirmed that IPCs provide similar improvement in symptoms and quality of life, and requires fewer hospital days, when compared against talc pleurodesis. Adverse events related to IPC treatment are uncommon and generally mild. Comparing the cost-effectiveness of IPC and pleurodesis is difficult and remains a contentious issue. Patients with a MPE are a heterogeneous group; it is likely some patients may benefit more from IPC and others from pleurodesis. How best to identify these subgroups is of high priority in MPE research. In the meantime, patients should be offered both options of IPC and pleurodesis (if no contraindication), and patient preference incorporated into the decision process.
\end{abstract}

Keywords Indwelling pleural catheter · Malignant pleural effusion · Pleurodesis · Thoracentesis · Cost-effectiveness . Complication $\cdot$ Talc

\footnotetext{
R. C. Boshuizen

Thoracic Oncology, the Netherlands Cancer Institute,

Amsterdam, the Netherlands

R. C. Boshuizen • R. Thomas • Y. C. G. Lee

Respiratory Medicine, Sir Charles Gairdner Hospital,

Perth, Australia

Y. C. G. Lee

School of Medicine \& Pharmacology and CAARR,

University of Western Australia, Perth, Australia

Y. C. G. Lee $(\bowtie)$

University Dept Med, 4/F G Blk, Sir Charles Gairdner Hospital,

Perth WA6009, Australia

e-mail: gary.lee@uwa.edu.au
}

\section{Introduction}

Malignant pleural effusion (MPE) is a common clinical problem with a growing incidence. Talc pleurodesis, first reported in 1935, remains a mainstay practice, testifying to a serious lack of progress in the clinical management in MPE. Studies in recent years have also raised concerns of the suboptimal efficacy and safety of talc pleurodesis [1-3].

Success of talc pleurodesis was often defined, in older literature, as no radiological evidence of fluid re-accumulation after 28-30 days, and reported as 90+\%. Large randomized studies, however, failed to reproduce such figures and instead showed a 30-day success of approximately $75 \%$, even in selected patients with no significant trapped lung. More importantly, the failure rate of talc pleurodesis increases the longer the patients survives; by six months only $50 \%$ patients still have adequate fluid control [4].

The advent of indwelling pleural catheters (IPCs) allows clinicians to question the wisdom of pleurodesis. The majority of patients with MPE have an incurable malignancy. The aim of management should be optimal palliation, especially against the associated breathlessness, which is often disabling, frightening and reduces quality of life $(\mathrm{QoL})$. The median survival for MPE patients is 3-4 months. Evacuation of the MPE often requires spending precious days of the patients' remaining life in hospital and can be associated with significant health care costs.

The ideal therapy for MPE patients should offer effective fluid control, improve dyspnea and QoL, be safe, minimally invasive and affordable and limit time in hospital.

\section{Advantages of Indwelling Pleural Catheter (IPC)}

The use of IPCs has grown exponentially, especially in the management of malignant pleural effusions, since the Food and Drug Administration approved their use in 1997. 
Commonly used commercially available devices include PleurX ${ }^{\circledR}$ catheter (CareFusion, USA) and Rocket ${ }^{\circledR}$ IPC (Rocket Medical, England), which have similar designs, and will hereafter be discussed generically as an IPC.

IPCs are 15.5 to 16 French silicone catheters and approximately $65 \mathrm{~cm}$ in length with a fenestrated section. The catheters are designed to remain in situ indefinitely for the remaining lifespan of the patient. The polyester cuff in the tunneled portion of the catheter promotes tissue fibrosis and avoids inadvertent catheter dislodgement. Tunneling of the catheter is believed to reduce infection risks, though this has not been formally tested. The one-way valve at the distal end of the catheter permits ambulatory drainage of pleural fluid: the catheter can be 'opened' and connected to drainage devices when needed, whilst at other times the patient can continue usual daily activities with the catheter capped off.

Insertion of the catheter is a minor intervention, usually requiring only local anesthesia or conscious sedation. It can be performed as an outpatient day procedure and the patient is ambulant promptly afterwards. Two incisions are made, between which the polyester cuff is tunneled into the subcutaneous tissue. The fenestrated part is inserted into the pleural space using Seldinger's technique via the cranial incision.

The frequency of drainage can be determined by the patient, depending on the rate of fluid re-accumulation and severity of symptoms, mainly dyspnea. It thus provides greater control and flexibility to the patients in managing their condition. Drainage itself can be performed by a trained carer or health care worker, removing the need of repeated invasive pleural drainage procedures, and the associated risks, costs and hospitalizations.

\section{Effective fluid control}

An estimated $50 \%$ of patients with a MPE will develop symptomatic re-accumulation requiring more definitive therapy to control fluid recurrence. Pleurodesis, either mechanically (via surgery), e.g. pleurectomy, or chemically using a sclerosing agent, has been the treatment of choice for decades. Surgical pleurodesis is costly, carries anesthetic risks and is associated with post-procedural intercostal neuralgia [5], and is not appropriate in the majority of MPE patients who have advanced malignancies with a limited life expectancy. Pleurodesis, especially via bedside instillation of a sclerosant, is the most common procedure worldwide to control MPE recurrence.

Talc has been shown to be the most cost-effective slerosant [6]. However, recent studies have confirmed that talc pleurodesis (either by thoracoscopic poudrage or instillation as a slurry) is effective in around $75 \%$ of cases at one month [4]. More importantly, the longer the patient survives, the more likely there will be fluid re-accumulation necessitating further interventions. In the largest randomized trial of MPE to date, only $50 \%$ of patients will still have adequate fluid control by 6 months post-talc pleurodesis [4]. The median survival of patients with a metastatic pleural carcinoma is around 4 months, and those with a primary pleural mesothelioma have a median survival about 9-12 months. Thus, the lifetime control of fluid re-accumulation by pleurodesis is suboptimal.

In addition, pleurodesis is only appropriate when the patient has full lung expansion after fluid drainage. The incidence of trapped lung varies among studies. Even for patients admitted to the hospital with the intent to perform pleurodesis, approximately $25 \%$ were found to be unsuitable, most often due to insufficient lung expansion, as shown in a study in the Netherlands $[2,7]$.

IPC is now accepted as the preferred management in patients with failed pleurodesis or in whom pleurodesis is inappropriate (e.g. trapped lung). IPCs function as a continuous access to the pleural cavity, through which pleural fluid can be drained without the need for repeat invasive procedures. A wealth of literature has testified to the effectiveness of IPCs in pleural fluid drainage. In most studies, over $90 \%$ of patients treated with an IPC do not require any further effusion-related pleural intervention $[8 \bullet, 9 \bullet, 10]$.

It is therefore logical to examine if IPC can be used as the frontline treatment in place of pleurodesis, rather than only when the latter is contraindicated or fails. Several key studies have now established that IPC has at least an equivalent benefit when compared with pleurodesis, thus challenging the conventional treatment paradigm [11] since the first randomized controlled trial comparing IPC with (doxycycline) pleurodesis was published in 1999 [12], including a UK multicenter randomized trial [8•], and a small study from the USA [13]. Additional randomized studies on the benefits of IPC over pleurodesis are also underway in the Netherlands and Australasia.

Two schools of thoughts exist in the clinical application of IPC. In many centers, it is advocated as an alternative to pleurodesis (see below), and a strong focus of the drainage regime is to keep the pleural cavity dry, e.g., via daily or alternate day fluid drainages [14-18]. Others believe that the goal of MPE management is symptom palliation, and evacuation of fluid should only need to be performed as guided by patient symptoms [19-21]. Often, complex drainage schedules are described [10, 12, 22-24].

In either case, spontaneous pleurodesis, if it occurs, is an added advantage as it allows removal of the catheter, thus negating the associated risks of IPC-related complications, inconvenience and costs. Spontaneous pleurodesis associated with IPC use has varying definitions in published literature (Fig 1). Rates of spontaneous pleurodesis range from $26 \%$ to $76 \%$ in most large series $[8 \cdot, 9 \bullet, 10,25,26]$, and the median time to spontaneous pleurodesis varies from 1 to 
Fig. 1 A literature summary of published rates of spontaneous pleurodesis in IPC patients, and the different definitions used

\section{$\operatorname{Re}$}

Ref 15

Ref 17

Ref 27

Ref 10

Ref 20

Ref 31

Ref 34

Ref 16

Ref 25

Ref 12

Ref 14

Ref 24

Ref 13

Ref 30

Total

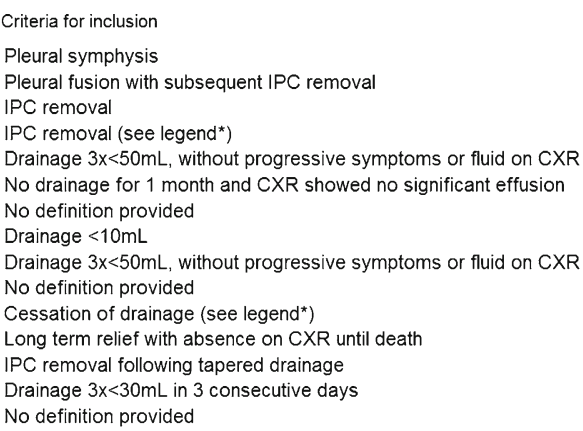

Incidence

$21 / 100$

$11 / 5$

$19 / 82$

$110 / 418$

$22 / 63$

$37 / 90$

$13 / 31$

$22 / 52$

$03 / 240$

$42 / 91$

$26 / 51$

$11 / 19$

$173 / 295$

$17 / 26$

$55 / 125$

$722 / 1734$

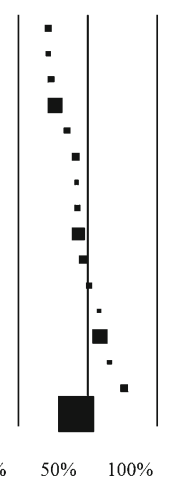

* the inclusion criteria of Ref 8 and 10 included no clinical or radiological re-accumulation of fluid, IPC removal, and no subsequent need for further pleural interventions
3 months [10, 12, 25]. Numerous factors may confound the incidence of pleurodesis, including the incidence of trapped lung in the study cohort, duration of patient survival, underlying types of malignancy and if pleurodesis has been attempted before [10].

The amount of fluid drained in the first week has also been suggested as a predictor of spontaneous pleurodesis, as the median drainage is significantly less in the group that achieves pleurodesis as compared to the group that does not [12]. Studies are underway to investigate the influence of drainage schedules on pleurodesis rates. Whether these different regimens will lead to reliably better pleurodesis rates in real world setting will also depend on the patient's compliance with the prescribed drainage schedules.

The rate of spontaneous pleurodesis may be lower in cases of lung cancer compared to other malignancies and in the presence of a trapped lung [24]. Even in patients unsuitable for talc pleurodesis who undergo IPC insertion, most patients have symptomatic benefit and spontaneous pleurodesis has been reported $[16,17]$.

\section{Quality of Life (QoL)}

It is increasingly realized by clinicians and patients that the primary goal of MPE management is palliation, and thus QoL is an essential measurement in assessing any therapy for MPE. However, studying QoL is difficult in this patient population. To date, there are no validated instruments specific for QoL assessment in MPE patients. The patients' sense of well-being is often influenced by numerous factors not directly related to the MPE. Confounding comorbidity from the underlying cancer and/or its treatment, as well as psychological issues such as uncertainty about prognosis, produce significant study 'noise' that hinders reliable assessment of the impact of MPE therapy in QoL of any individual patient.

The same difficulties apply even if the study endpoint is restricted to the impact of MPE therapy on dyspnea. Differential diagnosis of dyspnea in patients with end-stage cancer is broad and may not always be directly related to MPE alone. No dyspnea score has been validated for the use in patients suffering from MPE.
Withstanding these limitations, important attempts have been made to decipher the benefits of IPC management in QoL and symptom control.

In 1999, Putnam and colleagues showed in a randomized controlled trial with 144 patients (45 pleurodesis: 99 IPC) that at 30 days the modified Borg scale (MBS) dyspnea scores were significantly improved in the IPC group compared to those who received doxycycline pleurodesis [12].

A recent randomized study of $106 \mathrm{MPE}$ patients (54 pleurodesis: 52 IPC) employed the Visual Analog Scale (VAS) for dyspnea assessment. The study showed that IPC provided equivalent improvement in dyspnea compared with pleurodesis at 6 weeks after randomization [8•]. Dyspnea control at 6 months after randomization was superior in the IPC group. Quality of Life Questionnaire (QLQ-30) did not show significant differences at any time point between the two treatment arms.

Another randomized trial of 57 patients (29 pleurodesis: 28 IPC) employed multiple instruments for QoL assessment (distress score, number of symptoms, the Memorial Symptom Assessment Scale, changes in function form, performance status, dyspnea score, physical function, emotional state, and social life). Overall, patients randomized for IPC treatment had better dyspnea scores than those pleurodesed, especially in patients with trapped lungs [13].

Allowing patients the choice between IPC and pleurodesis may also provide a sense of control over their palliation treatment. In a non-randomized, patient-choice study, patients who chose to have IPC $(n=34)$ were significantly more likely to report improvement in QoL (recorded on a visual analog scale) than those who elected to receive talc pleurodesis $(n=31)$, $p=0.02$ [9•]. Interestingly, in this study when patients were given a choice, approximately equal number of patients chose IPCs and talc pleurodesis [9•].

Concluded from FACIT-TS-G questionnaires, the majority of patients treated with IPC was satisfied, would recommend it to others with MPE and would choose it again [27•]. In a non-randomized observational study (82 IPCs), patients reported significant improvements QoL and dyspnea assessed by QLQ-C30 and QLQ-LC13, at 2 and 14 weeks after IPC insertion [27•]. 
Despite the limitation of the study tools and the variations in trial protocols, the overall picture that has emerged from the published data would support that IPC is at least as efficient as (talc) pleurodesis in relieving dyspnea and/or improving QoL.

\section{Safe treatment for MPE}

The overall safety of IPC use has been confirmed in many observation series as well as randomized and non-randomized trials. Wrightson et al. summarized the reported complication rates in published literature [28] and showed that commonly reported adverse events are mild and easily controlled. Postinsertion pain was the most commonly reported side effect, affecting about one-third of patients, but is transient ( $<72$ hours) and usually required only simple analgesia. More significant complications, e.g. symptomatic loculation $(<10 \%)$, infection or catheter tract metastases, do occur, but at low and very acceptable incidences ( $<5 \%$ ) (Fig. 2).

Infections The fear of pleural infection from a long-term implanted device is the most significant deterring factor for the uptake of IPC. This concern is understandable, considering that most of these patients are elderly and with advanced malignancy (some of whom may undergo chemotherapy).

To date, all literature series suggest IPC infection rates are low. Morel and colleagues showed that seven out of 82 patients with IPCs developed a pleural infection [29]. The incidence rate was actually lower (one of 23) in patients treated with chemotherapy compared with six of 59 patients who did not receive chemotherapy (4\% vs $10 \%$ ). In the TIME-2 randomized study of 106 patients, five had a significant pleural infection, of which only one received chemotherapy. The risk of any IPC related pleural infection must also be analyzed in the context of the cumulative risks of alternative management, which often involves multiple pleural interventions (e.g. repeated drainages for trapped lung patients).
It is likely that infection was acquired during the course of the use of IPC, rather than at the time of insertion. Of 18 publications on IPC and pleural infections, only Sioris reported to have used antibiotic prophylaxis (Cephalexin, $750 \mathrm{mg}$ BID for 5 days) at the time of insertion, and showed an infection rate similar to other series [17]. As the majority of patients treated with IPC and chemotherapy do not develop pleural infections, routine prophylactic antibiotics (even during immunosuppressed phase) appear unwarranted.

Tract metastasis Fifteen tract metastases were reported in 13 studies with a combined number of nearly 1,000 IPC insertions [12, 14-17, 20, 25, 30-35]. Tract metastases are more often described in mesothelioma patients, but have been described for metastatic carcinomas as well. There is a suspicion that tract metastases occur more frequently the longer the IPC is in situ, though the incidence is too low to allow meaningful analyses [33]. Importantly, catheter tract metastases respond very promptly to radiotherapy, which can be performed with the IPC in situ.

The value of prophylactic radiotherapy in pleural puncture sites in mesothelioma patients remains a contentious issue [36]. The protective value of radiotherapy is even more doubtful in the setting of IPC as the risk of tumor invasion of the catheter tract is ongoing, which differs from the risks from one-off pleural interventions (such as thoracotomy) [36]. Thus, radiotherapy should be reserved for patients with symptomatic tract metastases only.

Mechanical defects Mechanical faults of the IPC drainage system can arise infrequently but most can be corrected without removal of the catheter. For example, catheter clogging occurs in a minority of cases and can be successfully treated with instillation of fibrinolytics. Rarely, mechanical defects (e.g. incompetent one-way valve) can occur, but most can easily be replaced [37]. Removal of IPC can be difficult and fracture of the catheter leaving residual portions
Fig. 2 A literature summary of published rates of (a) cellulitis and (b) empyema in IPC patients, showing a low incidence of both complications in all series

$\begin{array}{lc}\mathbf{a}_{\text {Ref }} & \text { Incidence } \\ \text { Ref } 10 & 4 / 418 \\ \text { Ref 24 } & 4 / 295 \\ \text { Ref 25 } & 4 / 250 \\ \text { Ref } 17 & 1 / 51 \\ \text { Ref } 31 & 2 / 90 \\ \text { Ref } 16 & 2 / 52 \\ \text { Ref } 14 & 1 / 24 \\ \text { Ref 27 } & 4 / 82 \\ \text { Ref 35 } & 1 / 17 \\ \text { Ref } 12 & 6 / 91 \\ \text { Ref } 8 & 6 / 52 \\ \text { Ref 32 } & 4 / 25 \\ \text { Ref 21 } & 2 / 11 \\ \text { Total } & 41 / 1458\end{array}$

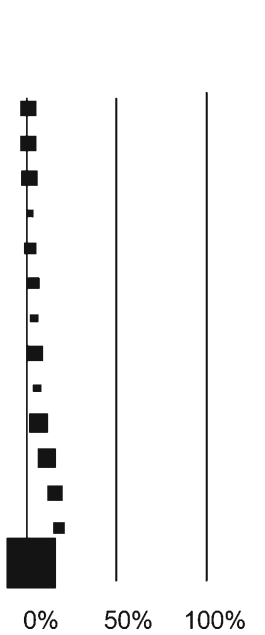

b

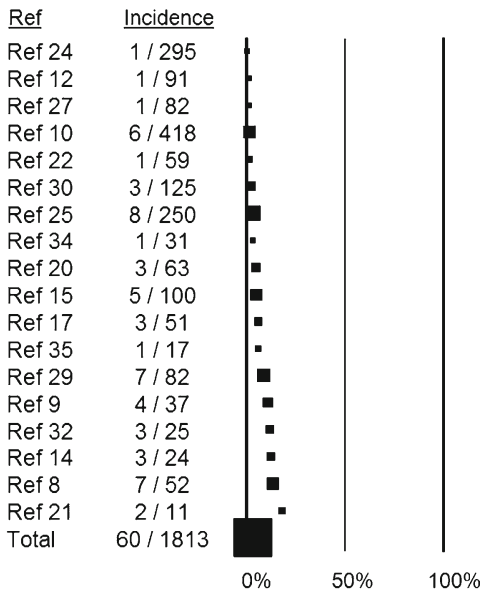


in situ has been reported. This should not raise concerns as the IPC is intended to remain for the remaining lifespan of the patient and retained segments have not been shown to increase morbidity [38].

\section{Reducing hospitalization}

Most patients with a MPE have advanced diseases and the prognosis is grave. Allowing patients to remain ambulatory outside hospital is one of the main goals in palliative care. IPCs are predominantly placed on an outpatient (or day case) basis, or at most requiring an overnight stay. Pleurodesis however requires inpatient care that can commonly take up about 6 days (the commonest quoted figure for talc slurry pleurodesis). Given a median survival of 3-4 months in patients suffering from a MPE, the hospital time for pleurodesis represents a significant proportion of the patients' remaining lifespan. When pleurodesis fails or is contraindicated, repeated pleural drainage requires further (often multiple) hospital admissions.

Hospitalization time for pleurodesis differs among centers, and with various methods of pleurodesis. Comparison of inpatient care time by pulled data from different studies is therefore inappropriate. Direct comparison of hospital days between patients treated with IPC and pleurodesis is the subject of a randomized trial in Australasia. It should be emphasized that it is the cumulative hospital admission days (till death) that matter most to the patient, not just the initial hospital stay for the IPC or pleurodesis.

Putnam and coworkers retrospectively reviewed hospital durations for patients with an inpatient chest drain $(n=68)$, as well as inpatient $(n=40)$ and outpatient $(n=60)$ indwelling pleural catheter. The latter group has significantly shorter hospitalization time (median 0 vs. 7 days) for the initial procedure [15].

In the patient choice study described before [22], patients who chose to receive an IPC spent significantly fewer days in hospital for admissions due to effusion-related reasons or overall hospital days from procedure till death (median $3 \mathrm{vs.}$ 10 days; $p<0.001$ and 6.5 vs. 18 days; $p=0.002$, respectively), as compared with the bedside pleurodesis group [9•]. In the TIME-2 study patients randomized to the IPC group spent a median of 1 day in hospital (vs. 4.5 days in the pleurodesis group, $p<0.001)$ for drainage or drain-related complications after 12 months [8•].

A recent pilot study also suggested that the insertion of an IPC immediately following thoracoscopic poudrage would decrease the hospital days significantly [23].

\section{Cost-effectiveness}

The cost-effectiveness of IPC (vs conventional pleurodesis) has provoked considerable discussions. No studies have performed thorough cost analyses comparing IPC and pleurodesis, in part because of the significant difficulties in such analyses. Most published works are retrospective or described only the costs of IPC (without direct head-on comparison with pleurodesis). Other papers rely on mathematical models comparing IPC against alternative treatments; the data have significant shortcomings and often cannot be extrapolated to other healthcare settings.

IPC and pleurodesis involved very different cost items and the quantity needed for each item varies widely among patients. There are also many factors such as survival, likelihood of success of pleurodesis, complication rates, etc., which either vary significantly in the literature and/or differ considerably among individual patients.

For example, IPC predominantly requires outpatient care, and most of the costs depend on the catheter and drainage kits, the latter depends on the frequency of drainage regime, which can vary from daily to as required only (see above). Whether the drainage is performed by a family member or requires home visits from community nurses, and how often the patient is reviewed by medical staff, will also drastically alter the costs. The duration of IPC drainage also varies widely depending on patient survival, and if spontaneous pleurodesis develop. Complications are uncommon, as discussed, but in those small proportion of patients the costs can rise sharply (e.g. inpatient for intravenous antibiotics for IPC related pleural infection). The longer the IPC is in situ, the more drainage devices the patient will consume, the higher the risks of complications and the more healthcare resources (e.g. community nurse visits) are needed.

On the other hand, pleurodesis costs vary significantly among centers, and the price for hospital bed days varies greatly around the world. Surgical pleurodesis is generally more expensive than bedside pleurodesis. If pleurodesis fails, subsequent costs will have to include additional intervention procedures.

In 2000, Putnam showed that early (7-day) hospital charges were lowest when IPC was inserted on an outpatient basis, compared with both inpatient IPC insertion and chest tube doxycycline pleurodesis. Total (long-term) hospital charges did not differ significantly between these groups; neither did overall survival [15].

Puri and colleagues compared four pleural interventions for MPE management in their cost-effective analysis: repeated thoracentesis, IPC, bedside pleurodesis and thoracoscopic pleurodesis. Based on projected figures the paper concluded that IPC is most cost-effective in providing Quality Adjusted Life Years (QALYs) for patients with limited (i.e. $<3$ months) survival. If the patient is to survive 12 months or more, bedside pleurodesis was the most economical [39].

Another study also showed that IPCs appeared to be more cost-effective in patients with a life expectancy of less than 6 weeks compared to those with longer survival. 
Effective treatment was defined as resolved effusion following survival of all potential life threatening complications caused by either IPC insertion or talc pleurodesis. Resolution of effusion was assumed to be higher from talc pleurodesis than IPC insertion (based on figures of $80 \%$ vs. $45 \%$, respectively). The IPC complication rate was estimated at $7.5 \%$, and $1.5 \%$ for talc pleurodesis. IPC induced pleural adherence was estimated to occur after 10 weeks, and patients were assumed to be visited by a nurse thrice weekly for drainage [40]. Caution must be exercised in adopting these conclusions as even small changes in the figures used for calculation (e.g. pleurodesis success rate) can profoundly alter the cost-effectiveness ratios [41] and the figures used for analysis are sometimes contestable.

Clinicians are also notoriously poor in predicting patient's life expectancy in MPE. For example, the CALGB study ( $n=482$ MPE patients) had a recruitment criterion of a life expectancy of at least 2 months; however, almost $50 \%$ of the recruited patients died within 30 days [4]. Treatment decisions based on prognosis may be difficult to implement until better means of assessing survival is available for MPE patients.

The concept of combined use of IPC as well as pleurodesis is now being explored, either by implanting IPC post-thoracoscopic pleurodesis [23], or to instill talc slurry via an IPC. Successful combination of both management strategies may help reduce costs.

The price of drainage catheters and bottles varies among countries and vendors, and are subjected to market competitions. A change in the pricing of these consumables will also dramatically alter the results of the cost-effectiveness assessments. Other forms of less expensive devices (such as reusable Surgivac pumps in Egypt) are also being tried in various centers, which will influence healthcare costing [42].

\section{Conclusion and future directions}

The use of IPC is gathering momentum around the world, more than a decade after it gained FDA approval. Its efficacy is well proven as a rescue treatment of MPEs when pleurodesis has failed or is contraindicated. In addition, the use of IPC as a frontline therapy in place of pleurodesis is growing in popularity. IPC provides improvements in QoL and dyspnea scores at least similar to talc pleurodesis. No major safety issues have been identified. IPCs significantly reduce pleural interventions, hospital admissions and inpatient expenses. The use of IPC also highlights the priorities of MPE management, i.e. improving symptoms and QoL, rather than to create pleural symphysis.

The precise benefits of IPC, especially in its costeffectiveness and impact on QoL, require further investigations. It is likely that different subsets of patients may benefit from using IPC or pleurodesis as first line of management for their MPE. Future studies are needed to help identify criteria to guide the choice of therapy in individual patients. Combining both pleurodesis and IPC treatments may further increase effectiveness [23]. In the meantime, patients should be offered both options of IPC and pleurodesis (if no contraindications) and patient preference should be incorporated into the decision process on clinical management.

Acknowledgements Prof Lee receives research grant funding from the Cancer Council of Western Australia, New South Wales Dust Disease Board, Westcare (Australia) and the Sir Charles Gairdner Research Advisory Committee.

Conflict of Interest Rogier C. Boshuizen is coinvestigator of a randomized trial comparing IPC with talc pleurodesis. Rocket Medical provided the majority of IPC and related equipment used in the trial without charge.

Rajesh Thomas declares no conflict of interest.

Y. C. Gary Lee has received honoraria from Carefusion Ltd and Sequana Medical Ltd as an advisory board member. Prof Lee was a coinvestigator of the TIME-2 study in which Rocket Ltd provided the equipments without charge.

\section{References}

Papers of particular interest, published recently, have been highlighted as:

- Of importance

1. Brant A, Eaton T. Serious complications with talc slurry pleurodesis. Respirology. 2001;6(3):181-5.

2. Burgers JA, Kunst PW, Koolen MG, et al. Pleural drainage and pleurodesis: implementation of guidelines in four hospitals. Eur Respir J. 2008;32(5):1321-7.

3. Lee YC, Light RW. Management of malignant pleural effusions. Respirology. 2004;9(2):148-56.

4. Dresler CM, Olak J, Herndon JE, et al. Phase III intergroup study of talc poudrage vs talc slurry sclerosis for malignant pleural effusion. Chest. 2005;127(3):909-15.

5. Brims FJ, Davies HE, Lee YC. Respiratory chest pain: diagnosis and treatment. Med Clin North Am. 2010;94(2):217-32.

6. Ong KC, Indumathi V, Raghuram J, et al. A comparative study of pleurodesis using talc slurry and bleomycin in the management of malignant pleural effusions. Respirology. 2000;5(2):99-103.

7. Boshuizen RC, Vincent AD, Kunst PW, et al. Talc instillation consensus AIDS differentiating successful from unsuccessful pleurodesis: a survey on the interpretation of pleural approximation after chest tube placement. Respiration. 2013;85(1):85-6.

8. - Davies HE, Mishra EK, Kahan BC, et al. Effect of an indwelling pleural catheter vs chest tube and talc pleurodesis for relieving dyspnea in patients with malignant pleural effusion: the TIME2 randomized controlled trial. JAMA. 2012;307(22):2383-9. A large randomized controlled trial comparing IPC as first line treatment for MPE. The study showed that IPC provided similar benefits in improving symptoms and quality of life as pleurodesis.

9. - Fysh ET, Waterer GW, Kendall P et al. Indwelling Pleural Catheters Reduce Inpatient Days over Pleurodesis for Malignant Pleural Effusion. Chest 2012. This non-randomized study allowed patient to choose between talc pleurodesis and IPC for management of MPE. Patients who chose to receive an IPC spent 
significantly fewer days in hospital from the time of pleural procedure to death (or end of follow-up).

10. Suzuki K, Servais EL, Rizk NP, et al. Palliation and pleurodesis in malignant pleural effusion: the role for tunneled pleural catheters. J Thorac Oncol. 2011;6(4):762-7.

11. Lee YC, Fysh ET. Indwelling pleural catheter: changing the paradigm of malignant effusion management. J Thorac Oncol. 2011;6 (4):655-7.

12. Putnam Jr JB, Light RW, Rodriguez RM, et al. A randomized comparison of indwelling pleural catheter and doxycycline pleurodesis in the management of malignant pleural effusions. Cancer. 1999;86(10):1992-9.

13. Demmy TL, Gu L, Burkhalter JE, et al. Optimal management of malignant pleural effusions (results of CALGB 30102). J Natl Compr Canc Netw. 2012;10(8):975-82.

14. Musani AI, Haas AR, Seijo L, et al. Outpatient management of malignant pleural effusions with small-bore, tunneled pleural catheters. Respiration. 2004;71(6):559-66.

15. Putnam Jr JB, Walsh GL, Swisher SG, et al. Outpatient management of malignant pleural effusion by a chronic indwelling pleural catheter. Ann Thorac Surg. 2000;69(2):369-75.

16. Qureshi RA, Collinson SL, Powell RJ, et al. Management of malignant pleural effusion associated with trapped lung syndrome. Asian Cardiovasc Thorac Ann. 2008;16(2):120-3.

17. Sioris T, Sihvo E, Salo J, et al. Long-term indwelling pleural catheter (PleurX) for malignant pleural effusion unsuitable for talc pleurodesis. Eur J Surg Oncol. 2009;35(5):546-51.

18. Stather DR, Tremblay A. Use of tunneled pleural catheters for outpatient treatment of malignant pleural effusions. Curr Opin Pulm Med. 2007;13(4):328-33.

19. Bertolaccini L, Zamprogna C, Barberis L, et al. Malignant pleural effusions: review of treatment and our experience. Rev Recent Clin Trials. 2007;2(1):21-5.

20. Cases E, Seijo L, Disdier C, et al. Use of indwelling pleural catheter in the outpatient management of recurrent malignant pleural effusion. Arch Bronconeumol. 2009;45(12):591-6.

21. Pien GW, Gant MJ, Washam CL, et al. Use of an implantable pleural catheter for trapped lung syndrome in patients with malignant pleural effusion. Chest. 2001;119(6):1641-6.

22. Hunt BM, Farivar AS, Vallieres E et al. Thoracoscopic Talc Versus Tunneled Pleural Catheters for Palliation of Malignant Pleural Effusions. Ann Thorac Surg 2012.

23. Reddy C, Ernst A, Lamb C, et al. Rapid pleurodesis for malignant pleural effusions: a pilot study. Chest. 2011;139(6):1419-23.

24. Warren WH, Kim AW, Liptay MJ. Identification of clinical factors predicting pleurx catheter removal in patients treated for malignant pleural effusion. Eur J Cardiothorac Surg. 2008;33(1):89-94.

25. Tremblay A, Michaud G. Single-center experience with 250 tunnelled pleural catheter insertions for malignant pleural effusion. Chest. 2006;129(2):362-8.
26. Tremblay A, Mason C, Michaud G. Use of tunnelled catheters for malignant pleural effusions in patients fit for pleurodesis. Eur Respir J. 2007;30(4):759-62.

27. - Sabur NF, Chee A, Stather DR et al. The Impact of Tunneled Pleural Catheters on the Quality of Life of Patients with Malignant Pleural Effusions. Respiration 2012. In this observational study, the authors demonstrate that patients would recommend an IPC to others and would choose it again.

28. Wrightson JM, Fysh E, Maskell NA, et al. Risk reduction in pleural procedures: sonography, simulation and supervision. Curr Opin Pulm Med. 2010;16(4):340-50.

29. Morel A, Mishra E, Medley L, et al. Chemotherapy should not be withheld from patients with an indwelling pleural catheter for malignant pleural effusion. Thorax. 2011;66(5):448-9.

30. Bazerbashi S, Villaquiran J, Awan MY, et al. Ambulatory intercostal drainage for the management of malignant pleural effusion: a single center experience. Ann Surg Oncol. 2009;16(12):3482-7.

31. Bertolaccini L, Viti A, Gorla A et al. Home-management of malignant pleural effusion with an indwelling pleural catheter: Ten years experience. Eur J Surg Oncol 2012.

32. Burgers JA, Olijve A, Baas P. Chronic indwelling pleural catheter for malignant pleural effusion in 25 patients. Ned Tijdschr Geneeskd. 2006;150(29):1618-23.

33. Janes SM, Rahman NM, Davies RJ, et al. Catheter-tract metastases associated with chronic indwelling pleural catheters. Chest. 2007;131(4):1232-4.

34. Pollak JS. Malignant pleural effusions: treatment with tunneled long-term drainage catheters. Curr Opin Pulm Med. 2002;8 (4):302-7.

35. van den Toorn LM, Schaap E, Surmont VF, et al. Management of recurrent malignant pleural effusions with a chronic indwelling pleural catheter. Lung Cancer. 2005;50(1):123-7.

36. Davies HE, Musk AW, Lee YC. Prophylactic radiotherapy for pleural puncture sites in mesothelioma: the controversy continues. Curr Opin Pulm Med. 2008;14(4):326-30.

37. Bower C, Mahmood K. Re: noninvasive repair of broken tunneled pleural catheters. J Vasc Interv Radiol. 2011;22(2):255-6.

38. Fysh ET, Wrightson JM, Lee YC, et al. Fractured indwelling pleural catheters. Chest. 2012;141(4):1090-4.

39. Puri V, Pyrdeck TL, Crabtree TD et al. Treatment of Malignant Pleural Effusion: A Cost-Effectiveness Analysis. Ann Thorac Surg 2012.

40. Olden AM, Holloway R. Treatment of malignant pleural effusion: PleuRx catheter or talc pleurodesis? a cost-effectiveness analysis. J Palliat Med. 2010;13(1):59-65.

41. van den Berg M, Hamberg-van Reenen HH, Schoemaker CG. Cost-effectiveness: Euros per QALY is not enough. Ned Tijdschr Geneeskd. 2012;156(5):A3817.

42. Al-Halfawy A, Light R. Safety and efficacy of using a surgivac pump for the drainage of chronic indwelling pleural catheters in malignant pleural effusions. Respirology. 2008;13(3):461-4. 